\title{
Desenvolvimento e Caracterização de Compósitos Poliméricos à Base de Poli(Ácido Lático) e Fibras Naturais
}

\author{
Alessandra Luiza de Lemos \\ Artecola Indústrias Químicas Ltda., Campo Bom, RS \\ Ricardo Martins de Martins \\ Universidade Feevale, Novo Hamburgo, RS
}

\begin{abstract}
Resumo: O presente trabalho busca avaliar o comportamento de compósitos que contêm em sua formulação um biopolímero originário de indústrias da agricultura sustentável. O biopolímero selecionado, poli(ácido lático), (PLA) e um polímero termoplástico (poliuretano), (TPU), foram misturados a fibras naturais (madeira e coco). Em um primeiro momento, foram preparadas formulações contendo 10, 20 e 30\% em massa de fibra vegetal em relação à blenda de PLA-TPU (na proporção de 70/30\% em massa). Em um segundo momento, manteve-se a mesma proporção em massa de fibra vegetal em relação à blenda de PLA-TPU, porém foram adicionados $5 \%$ em massa de anidrido maleico. Na sequência, as formulações foram avaliadas através de caracterizações físico-mecânicas, térmicas e microscópica. De um modo geral, as análises mostraram evidências bastante conclusivas sobre a baixa adesão interfacial entre a blenda polimérica e as fibras vegetais. Deve-se salientar a fraca atuação do anidrido maleico como agente de acoplamento, haja vista que as formulações que o continham não apresentaram melhoras em suas propriedades. Portanto, os resultados indicam que os biocompósitos formulados possam atender à necessidade de nichos específicos de mercados populares, como o de decoração, os quais não demandam alto desempenho de seus produtos, viabilizando o uso de fibras vegetais devido à redução de custo do compósito final.
\end{abstract}

Palavras-chave: Biopolímero, poli(ácido lático), compósitos poliméricos, fibras vegetais.

\section{Development and Characterization of Polymeric Composites Based on Poly (Lactic Acid) and Natural Fibers}

\begin{abstract}
This work evaluates the behavior of composites containing a biopolymer derived from sustainable agriculture industries. The biopolymer selected, poly(lactic acid) - PLA -, in combination with a thermoplastic polymer (polyurethane) - TPU - was mixed with natural fibers, wood and coconut. In a first step, formulations containing 10, 20 and $30 \%$ by weight of fiber in relation to the blend of PLA-TPU (in the ratio 70/30 wt \%) were prepared. In the second step, the same set of formulations was maintained but with addition of $5 \mathrm{wt} \%$ of the coupling agent maleic anhydride. These composites were characterized by means of physical-mechanical, thermal and microscopic techniques. The results indicated a low interfacial bonding between the polymeric blend and the vegetal fibers. It is important to emphasize the poor performance of the coupling agent, because the formulations containing maleic anhydride did not show any improvement in their properties in comparison to the standard material. Therefore, the biocomposites studied in this paper can be used when high-performance materials are not required, as in home decoration articles, making it feasible to use vegetal fibers in order to reduce the cost of the final composite.
\end{abstract}

Keywords: Biopolymer, poly(lactic acid), polymeric composites, vegetal fibers.

\section{Introdução}

Muito tem sido discutido sobre as principais políticas de gestão de resíduos de materiais utilizados na indústria em geral, porém, a maior reflexão atual é sobre o desenvolvimento sustentável. A necessidade de viabilizar a continuidade do desenvolvimento, racionalizar o consumo, optar por soluções e produtos sustentáveis em todo o seu ciclo de vida e buscar o equilíbrio entre o homem e a natureza passaram a ser considerações fundamentais no desenvolvimento de produtos. Diversos materiais têm sido pesquisados em busca de soluções ambientalmente favoráveis, como podem ser apreciados em alguns trabalhos dispostos na literatura $^{[1-5]}$. Dentro deste contexto e como alternativa aos polímeros convencionais, os biocompósitos têm alcançado posições de destaque.

Intensas pesquisas estão sendo realizadas para o desenvolvimento desta nova classe de compósitos verdes (biocompósitos), combinando fibras naturais com polímeros biodegradáveis ${ }^{[1,5]}$. O maior destaque destes biocompósitos é o caráter ecologicamente correto, totalmente degradáveis e sustentáveis. No final de seu ciclo de vida, podem ser descartados ou sofrerem compostagem sem prejudicar o ambiente ${ }^{[6]}$. Deve-se, no entanto, salientar que os compósitos estudados neste

Autor para correspondência: Ricardo Martins de Martins, Universidade Feevale, Rod. RS 239, 2755, CEP 93352-000,

Novo Hamburgo, RS, Brasil, e-mail: ricardomartins@feevale.br 
trabalho não são totalmente biodegradáveis, haja vista que apresentam em sua formulação um polímero que não é caracterizado por sua biodegradabilidade, o poliuetano.

Um grande número desses polímeros biodegradáveis está disponível comercialmente ${ }^{[7]}$. Tais compostos mostram uma grande variedade de propriedades, podendo competir com os polímeros não-biodegradáveis em diferentes campos industriais (por exemplo, embalagens). Os biocompósitos têm sido utilizados de forma eficaz em muitas aplicações como produtos de consumo com ciclos de vida curtos ou produtos destinados a pouco tempo de uso antes do descarte apropriado ${ }^{[6,8]}$.

A utilização de reforço em materiais compósitos está se tornando cada vez mais comum no mercado, pois originam materiais com propriedades melhores $\mathrm{e}$ adequadas a uma determinada aplicação ${ }^{[9]}$. O emprego de fibras vegetais, além de ajudar na fragmentação dos compostos poliméricos descartados, reduz a quantidade de material polimérico utilizado na fabricação dos $\operatorname{mesmos}^{[5]}$.

O horizonte de utilização das fibras naturais é bastante amplo, abrangendo desde as aplicações clássicas na indústria têxtil até o reforço de matrizes poliméricas termoplásticas e termofixas. As fibras vegetais são menos abrasivas que as fibras inorgânicas usualmente utilizadas como reforço (por exemplo, fibra de vidro) e assim geram menor desgaste dos equipamentos envolvidos em seu processamento $^{[10,11]}$

Tanto a pesquisa acadêmica quanto as indústrias exploram novas formas de desenvolver produtos químicos mais ecologicamente corretos para uma variedade de aplicações. As fibras naturais oferecem a possibilidade de entregar maior valor agregado ao produto final em razão da diminuição de custos de sua fabricação, sustentabilidade e renovação, especialmente na indústria automotiva, além de apresentarem um impacto ambiental reduzido durante o processamento ${ }^{[12]}$. Resíduos agrícolas, como palhas de trigo e de arroz, bagaço e talos de milho também são fontes de origem vegetal, embora as suas fibras tenham menor teor de celulose em comparação à madeira $^{[13]}$.

Na seleção de fibras para reforço em compósitos, é imprescindível a consideração de vários fatores como: custo e disponibilidade, efeito sobre as características de fluidez do polímero, propriedades físicas, estabilidade térmica, resistência química, abrasividade ou desgaste, biodegradabilidade, toxicidade, reciclabilidade, molhabilidade e compatibilidade com a matriz polimérica ${ }^{[14-16]}$. Deve-se considerar, também, a possibilidade de ocorrência de incompatibilidade entre a matriz polimérica e a fibra, haja vista que a interação interfacial é, em muitos casos, muito fraca ${ }^{[17,18]}$. Nesse caso, faz-se uso de um terceiro componente no compósito, diminuindo a tensão interfacial e aumentando a adesão entre as fases da blenda polimérica: o agente compatibilizante. Há vários compostos que apresentam essa função, tais como copolímeros ${ }^{[19]}$, metacrilato de glicidila ${ }^{[20]}$, anidrido maleico, ${ }^{[21]}$ entre outros ${ }^{[19-21]}$. No que tange aos métodos de compatibilização de blendas poliméricas, os mesmos podem ser classificados como mecânicos, físicos e químicos. O primeiro consiste na obtenção de uma morfologia da blenda polimérica relativamente estável, que permita uma melhoria das propriedades da blenda através de fixação mecânica de uma fase na outra. Por sua vez, a compatibilização física é alcançada, geralmente, através do uso de copolímeros, cuja ação baseia-se na dissolução destes entre as fases da blenda. Por último, tem-se o método químico, no qual a compatibilização ocorre através de reações químicas que propiciem a formação de ligações químicas covalentes entre as fases da blenda.

Assim, este trabalho visa estudar e avaliar o comportamento dinâmico-mecânico, térmico e microscópico de compósitos que contêm em sua formulação o poli(ácido lático) (PLA), um bioplímero oriundo de indústrias que produzem e comercializam produtos sustentáveis para os setores agrícolas e industriais $^{[22]}$, assim como um poliuretano termoplástico (TPU), largamente empregado como elastômero de alto desempenho em uma variedade de aplicações, ${ }^{[23]}$ e a associação destes polímeros com fibras naturais de madeira e coco. Adicionalmente, utilizou-se anidrido maleico em algumas formulações como agente compatibilizante, em razão de seu relativo baixo custo comercial.

\section{Experimental}

\section{Materiais empregados e identificação dos compósitos}

Foram utilizados dois polímeros termoplásticos, dois tipos de fibras vegetais e um agente compatibilizante cujas características encontram-se a seguir:

- Poli(ácido lático) (PLA) (2002D): contém 4\% D-lactídeo, 96\% L-lactídeo; $\mathrm{M}_{\mathrm{w}}$ : $121.400 \mathrm{~g} / \mathrm{mol}$; MFI: $5-7 \mathrm{~g} / 10 \mathrm{~min}\left(210^{\circ} \mathrm{C}-2,16 \mathrm{~kg}\right)$; densidade: 1,24 ; forma física: grão; $\mathrm{Tg}: 60^{\circ} \mathrm{C}$, fornecido pela empresa Natureworks ${ }^{\mathrm{TM}}$;

- Poliuretano termoplástico (TPU) (contendo aproximadamente $60 \%$ de fonte renovável): MFI: 3-5 $\mathrm{g} / 10 \mathrm{~min}\left(210^{\circ} \mathrm{C}-2,16 \mathrm{~kg}\right)$; densidade: 1,13 ; forma física: grão; $\mathrm{Tg}$ : $-48^{\circ} \mathrm{C}$, fornecido pela empresa Merquinsa;

- Anidrido maleico (com teor mínimo de 99,5\% em massa): densidade: 1,47; forma física: pó; obtido da empresa Elekeiroz;

- Fibra de Madeira (Pinus): granulometria (em $\mu \mathrm{m})$ : $500(1,00 \%) ; 355(34,00 \%) ; 250(37,00 \%) ; 177$ (20,00\%); 90 (6,50\%); 45 (1,50\%); fundo $(0,00 \%)$; umidade: $7 \%$; fornecida pela empresa Inbrasfama;

- Fibra de Coco: granulometria (em $\mu \mathrm{m})$ : 500 $(39,50 \%) ; 355 \quad(44,00 \%) ; 250 \quad(13,50 \%) ; 177$ $(2,40 \%) ; 90(0,60 \%) ; 45(00,00 \%)$; fundo $(0,00 \%)$; umidade: $12 \%$; fornecida pela empresa Inbrasfama.

As amostras foram preparadas, em uma primeira etapa, com 10, 20 e $30 \%$ em massa de fibra vegetal em relação à blenda de PLA-TPU (na proporção de 70/30\% em massa). Na segunda etapa, manteve-se a relação de 10,20 e $30 \%$ em massa de fibra vegetal em relação à blenda de PLA-TPU (na proporção de 70/30\% em massa), porém adicionaram-se $5 \%$ em massa do agente de acoplamento - anidrido maleico.

$\mathrm{Na}$ Tabela 1, encontram-se os percentuais da blenda, fibra vegetal e anidrido maleico, em massa, para cada 
Tabela 1. Formulações avaliadas com fibras de madeira ( $\mathrm{FM}_{-}{ }_{-}$) ou de coco $\left(\mathrm{FC}_{-}{ }_{-}{ }_{-}\right)$.

\begin{tabular}{lccccccc}
\multicolumn{1}{c}{ Formulações (\%) } & Padrão & -_10SA & -_10CA & -_20SA & -_20CA & $\ldots$ 30SA & $\ldots$ 30CA \\
\hline PLA & 70,0 & 63,0 & 60,5 & 56,0 & 53,5 & 49,0 & 46,5 \\
TPU & 30,0 & 27,0 & 24,5 & 24,0 & 21,5 & 21,0 & 18,5 \\
Fibra Madeira (FM) & - & 10,0 & 10,0 & 20,0 & 20,0 & 30,0 & 30,0 \\
Fibra Coco (FC) & - & 10,0 & 10,0 & 20,0 & 20,0 & 30,0 & 30,0 \\
Anidrido Maleico & - & - & 5,0 & - & 5,0 & - & 5,0 \\
\hline
\end{tabular}

amostra de 500 gramas, sendo: FM - fibra de madeira; FC - fibra de coco; SA - sem anidrido maleico; CA - com anidrido maleico; 10/20/30 - os números representam a porcentagem em massa de fibra. Deve-se salientar que as formulações não apresentam mistura das duas fibras.

\section{Processamento}

As formulações foram preparadas a partir da medida particular de seus componentes com posterior mistura e homogeneização manual dos mesmos. Após, as referidas formulações sofreram o processo de extrusão em uma extrusora dupla rosca corrotante de laboratório, modelo CDR 22 - LD 40, da empresa Extrusão Brasil. As temperaturas nas diferentes zonas de aquecimento variaram segundo o seguinte perfil: $130-150^{\circ} \mathrm{C}$, $130-150^{\circ} \mathrm{C}, \quad 140-160^{\circ} \mathrm{C}, \quad 150-170^{\circ} \mathrm{C}, \quad 160-170^{\circ} \mathrm{C}$, entre as zonas 1 e 5 , respectivamente, $170-180^{\circ} \mathrm{C}$ no cabeçote e rotação de 30-70 rpm. Após a extrusão, os compostos obtidos, em formatos de fitas, foram préaquecidos $\left(100^{\circ} \mathrm{C}\right)$ em uma prensa quente (Hidraumak) por $1 \mathrm{~min}$, em seguida prensados por $30 \mathrm{~s}$, sob pressão de $50 \mathrm{kgf} / \mathrm{cm}^{2}$. Na sequência, foram resfriados em uma prensa (Eletrovale) em temperatura ambiente, sob pressão de $5 \mathrm{kgf} / \mathrm{cm}^{2}$ durante $30 \mathrm{~s}$. Desta forma, após esse processo, os corpos de prova foram confeccionados para as análises.

\section{Caracterizações}

Ensaio de resistência à tração, alongamento e módulo de elasticidade

O ensaio foi realizado segundo a norma ISO 527-4:1997. Foi utilizada uma máquina universal de ensaios EMIC, modelo DL 500 BF com célula de força de $500 \mathrm{kgf}$ e uma taxa de $5 \mathrm{~mm} / \mathrm{min}$. São registradas a força e a deformação, de forma que se possam calcular a tensão, o alongamento e o módulo de elasticidade.

\section{Ensaio de resistência à flexão}

O procedimento foi realizado de acordo com a norma ISO 178:2001. A flexão foi feita em três pontos de apoio. $\mathrm{O}$ ensaio foi realizado flexionando-se o corpo de prova a uma velocidade constante, e registrava-se a força versus a flecha de deformação.

A flexão das amostras foi determinada pela máquina universal de ensaios EMIC, modelo DL 500BF, com célula de carga de $500 \mathrm{kgf}$ e uma taxa de $10 \mathrm{~mm} / \mathrm{min}$.

Ensaio de determinação da temperatura de deflexão - HDT

O ensaio para determinação da temperatura de deflexão HDT foi realizado seguindo a norma ISO 75:2004, tendo sido executado em um equipamento HDT/VICAT Júnior modelo Ceast. Os corpos de prova foram cortados em formatos retangulares de $5 \times 1 / 2$ polegada. Aguardou-se a estabilização da temperatura em aproximadamente $38^{\circ} \mathrm{C}$ (temperatura programada). Após esta etapa, iniciou-se a análise através da leitura da temperatura de amolecimento no display digital.

Ensaio de caracterização térmica por calorimetria exploratória diferencial (DSC)

As análises foram realizadas em equipamento da marca Perkin Elmer, modelo Jade DSC e utilizado software Pyris Series para o tratamento dos dados. A faixa de temperatura da análise está descrita a seguir: primeiro aquecimento de $25^{\circ} \mathrm{C}$ a $200^{\circ} \mathrm{C}$ a uma taxa de $10^{\circ} \mathrm{C}$ por minuto. Após, um patamar isotérmico de $200^{\circ} \mathrm{C}$ por um minuto; na sequência, resfriamento de $200^{\circ} \mathrm{C}$ a $25^{\circ} \mathrm{C}$ a uma taxa de $10^{\circ} \mathrm{C} / \mathrm{min}$. Em seguida, novamente um patamar isotérmico por 1 minuto, porém a $25^{\circ} \mathrm{C}$; novo aquecimento até $200^{\circ} \mathrm{C}$ a uma taxa de $10^{\circ} \mathrm{C} / \mathrm{min}$. Após, 1 minuto a $200^{\circ} \mathrm{C}$, seguido de um segundo resfriamento até $25^{\circ} \mathrm{C}$ a uma taxa de $10^{\circ} \mathrm{C} / \mathrm{min}$, utilizando-se uma massa de amostra entre 2 a $5 \mathrm{mg}$.

\section{Ensaio de microscopia eletrônica de varredura (MEV)}

A adesão interfacial fibra/matriz foi avaliada em corpos de prova das amostras fraturadas no ensaio de tração (seção transversal das amostras), através de medidas por microscopia eletrônica de varredura (MEV, Jeol, JSM-6360LV), com uma tensão de aceleração de $5 \mathrm{kV}$ e deposição de uma fina camada de ouro por 3 minutos através de um metalizador.

\section{Resultados e Discussão}

Os ensaios de resistência à tração (Figura 1) demonstraram similaridade em relação a este parâmetro com o uso de 10 e $20 \%$ em massa de fibra, como também evidenciado por Zhao et al. ${ }^{[24]}$, e um acentuado aumento quando adicionados $30 \%$ em massa de fibra de madeira em relação à blenda polimérica padrão (sem adição de fibra). O mesmo comportamento foi verificado com as formulações as quais utilizaram o agente de acoplamento. No que tange às formulações contendo fibra de coco, as mesmas apresentaram desempenho abaixo daquele atribuído à blenda padrão. Em razão da falta de material, não foi possível avaliar o desempenho da formulação FC30SA referente ao presente ensaio.

A adição de fibras naturais à blenda polimérica mostrou, através dos resultados de tração, uma diminuição na resistência mecânica dos biocompósitos formados, quando comparados à blenda pura. Infere-se que a presença das fibras curtas dispersas na matriz 
polimérica pode ter produzido descontinuidades na mesma, dificultando a distribuição e transferência do esforço aplicado e, assim, reduzindo a tensão máxima suportada pelos biocompósitos desenvolvidos. Ademais, a orientação das fibras também influencia bastante nas propriedades dos compósitos, principalmente em fibras curtas, a qual a mudança de orientação é impossível de se prever. A orientação das fibras muda em razão de vários fatores como propriedades geométricas das mesmas, comportamento viscoelástico do polímero, entre outros $^{[8,25]}$.

No que concerne ao agente de acoplamento, é perceptível que sua inclusão nas formulações tornou-as menos resistentes à tração, quando se comparam os sistemas com e sem a presença de anidrido maleico. Tais resultados são um reflexo da fraca adesão que o anidrido proporcionou entre a matriz e as fibras, como poderá ser visto ao longo deste estudo.

$\mathrm{O}$ decréscimo de alongamento de ruptura (Figura 2) ocorreu de forma gradual, à medida que o percentual em massa de fibra natural aumentou, tendo drástica redução em $30 \%$ de fibra em massa para as formulações contendo fibra de madeira e fibra de coco sem anidrido.

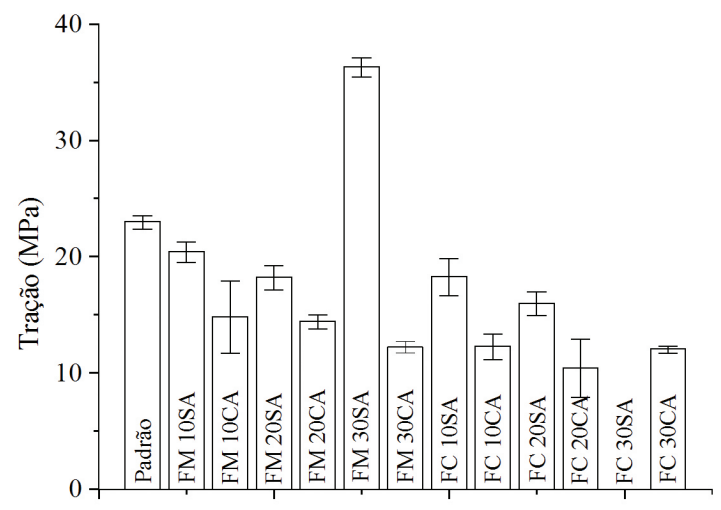

Formulações

Figura 1. Resultados do ensaio de tração para as formulações contendo fibras de madeira e coco na presença ou ausência de anidrido maleico.

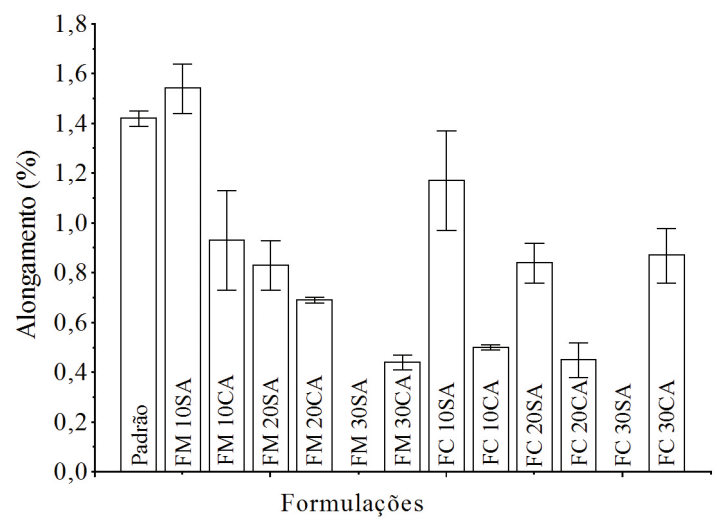

Figura 2. Resultados do ensaio de alongamento para as formulações contendo fibras de madeira e coco na presença ou ausência de anidrido maleico.
Por sua vez, observou-se o aumento do módulo elástico (Figura 3), conforme o aumento do reforço no compósito (formulação FM30CA). Este comportamento pode ser atribuído ao fato de que fibras com maior rigidez que a matriz podem aumentar o módulo de elasticidade, embora, geralmente, diminuem o alongamento ${ }^{[10]}$, indo ao encontro dos resultados obtidos na Figura 2. Tal ocorrência foi evidenciada tanto nas formulações com fibras de madeira quanto nas formulações com fibras de coco.

A inclusão de uma fase rígida, tal como fibras de madeira, é capaz de aumentar a rigidez da blenda polimérica $^{[8,25-28]}$. A fibra vegetal é um material lignocelulósico polar hidrofílico, que possui grupos hidroxila $(-\mathrm{OH})$ na superfície enquanto que a matriz polimérica poliolefínica é um material hidrocarbônico apolar hidrofóbico; e a adesão entre essas duas partes é auxiliada pelas formas e teores de lignina presentes nas fibras que pode variar de fibra para fibra e depende também da época da colheita, forma de secagem e tipo de tratamento ${ }^{[29]}$. Nesse ponto, devem ser referenciados os relevantes trabalhos de Barreto et al. ${ }^{[15,16,25,30]}$ acerca do uso de diversas fibras naturais para a síntese de biocompósitos. Os autores demonstraram, entre outros pontos, a importância do tratamento superficial no que tange a modificar as propriedades dos biocompósitos, justamente pela modificação do teor de lignina nas fibras através do uso de soluções alcalinas de hidróxido de sódio junto a hipoclorito de sódio. Com base nos estudos, concluíram que o tratamento químico superficial depende de vários fatores, tais como a concentração dos reagentes, temperatura, etc., e que o referido tratamento pode afetar de forma decisiva as propriedades térmicas, mecânicas e de adesão entre a matriz polimérica e as fibras. Analogamente, as diferenças entre os resultados encontrados neste trabalho podem estar relacionadas a diferentes teores de lignina nas fibras, aliado ao fato de a fibra de coco ser relativamente mais higroscópica do que a fibra de madeira, resultando em uma adesão mais fraca entre a fibra vegetal e a matriz.

Assim como no ensaio de resistência à tração (Figura 1), os resultados de flexão (Figura 4) são muito sensíveis à adesão interfacial fibra/matriz, o que não

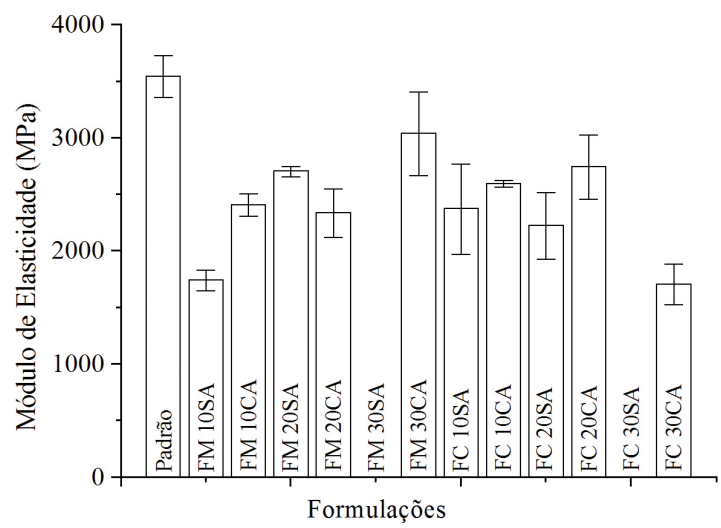

Figura 3. Resultados do ensaio do módulo de elasticidade para as formulações contendo fibras de madeira e coco na presença ou ausência de anidrido maleico. 


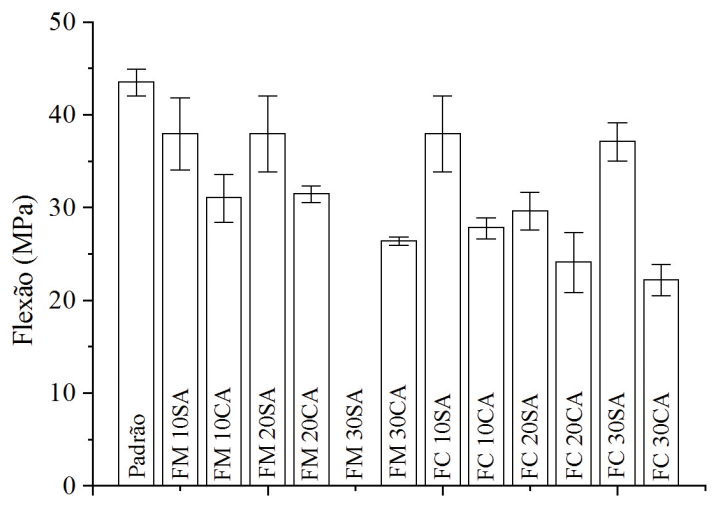

Formulações

Figura 4. Resultados do ensaio de flexão para as formulações contendo fibras de madeira e coco na presença ou ausência de anidrido maleico.

ocorre com o módulo (Figura 3). De fato, a interface desempenha um papel crucial na transferência da tensão da matriz à fase fibrosa. O reforço em matrizes poliméricas com fibras naturais é muitas vezes atribuído a uma forte adesão gerada através de interações de diferentes fases. As diferentes propriedades das superfícies das fibras (altamente polares) e das matrizes poliméricas apolares (hidrofóbicas) exigem uma estratégia adequada para melhorar a compatibilidade e adesão interfacial entre as $\operatorname{mesmas}^{[9,27,31]}$.

No que concerne à resposta mecânica dos compósitos, quando uma carga externa é aplicada aos mesmos, tal carga é transferida para as fibras mais próximas da superfície e continua fibra a fibra através da matriz e interface. Então, uma interface fraca induz uma distribuição de carga ineficaz e o efeito potencial de reforço das fibras continua inexplorado. Mas, uma interface forte pode garantir uma transferência eficiente da carga aplicada para reforços fibrosos através da matriz com consequente melhora do comportamento mecânico do composto ${ }^{[10,27]}$.

Através da análise dos resultados, não se verificou um aumento na resistência à flexão nos biocompósitos desenvolvidos à medida que a proporção das fibras vegetais foi sendo aumentada, conforme descrevem alguns trabalhos ${ }^{[5,9,10,29]}$. Tal comportamento sugere uma baixa adesão entre as fases da fibra e da matriz polimérica, corroborado pelo fato de a resistência à tração dos biocompósitos ter diminuído com o aumento da proporção de fibras ${ }^{[29]}$.

Qualitativamente, os resultados de flexão assemelham-se aos de tração no que se refere à diminuição do desempenho da propriedade analisada quando da adição de fibras. Os resultados de MEV (ver adiante) podem ajudar a compreender melhor tais resultados, haja vista que em ambos os ensaios de flexão e tração, o parâmetro analisado apresentou diminuição da propriedade em relação ao valor da blenda padrão. Não foi possível avaliar o desempenho da formulação FM30SA devido à falta de material para este ensaio.

Com o objetivo de verificar o comportamento do material no que concerne à deformação do mesmo sob flexão quando este é submetido a um aquecimento à

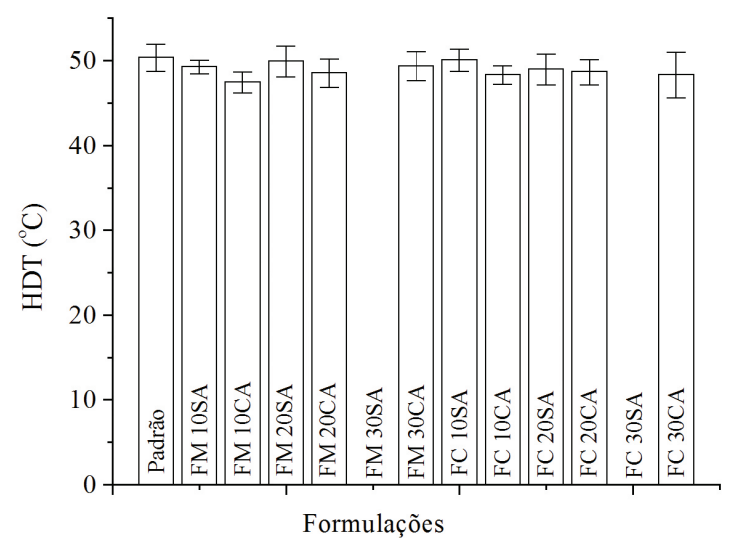

Figura 5. Resultados do ensaio de temperatura de deflexão para as formulações contendo fibras de madeira e coco na presença ou ausência de anidrido maleico.

taxa constante, realizou-se o ensaio de temperatura de deflexão (HDT) ${ }^{[32]}$, mostrado na Figura 5.

O ensaio deflexão térmica HDT apresentou valores de temperatura menores do que a blenda pura, à medida que a porcentagem de fibra foi aumentada, mostrando que as fibras vegetais ocasionaram sensível aumento na deflexão térmica dos biocompósitos e uma melhora no desempenho de penetração, em relação à matriz polimérica pura. Concomitantemente, os resultados evidenciaram que não houve um aumento na estabilidade dimensional em temperaturas mais elevadas sem a ocorrência de distorção térmica da peça ${ }^{[33]}$. Isso pode estar associado à molhabilidade das fibras ${ }^{[28,34]}$. Uma vez que a análise dos ensaios de tração e flexão apresentou resultados abaixo daqueles relacionados à blenda padrão, a tendência dos resultados de temperatura HDT vai ao encontro dos dados da literatura ${ }^{[35]}$. Não foi possível avaliar o desempenho das formulações FM30SA e FC30SA devido à falta de material para este ensaio.

Partindo-se, agora, para a caracterização térmica dos sistemas aqui estudados, a Tabela 2 apresenta os dados da análise de DSC obtidos através das curvas de DSC (não mostradas) das formulações. Na referida tabela, têm-se a temperatura de transição vítrea $(\mathrm{Tg})$, a temperatura de cristalização (Tc), a entalpia de cristalização $\left(\Delta \mathrm{H}_{\mathrm{c}}\right)$, a temperatura de fusão (Tm), a entalpia de fusão $\left(\Delta H_{m}\right)$ e o grau de cristalinidade $(\mathrm{Xc})$ dos compostos.

A temperatura de transição vítrea (Tg) típica do PLA varia de $50^{\circ} \mathrm{C}$ a $80^{\circ} \mathrm{C}$, enquanto que sua temperatura de fusão (Tm) varia de $130^{\circ} \mathrm{C}$ a $180^{\circ} \mathrm{C}^{[36]}$.

Percebe-se, pela análise da Tabela 2, que a inclusão da fibra vegetal, na presença ou não do agente compatibilizante, pouco influenciou a temperatura de transição vítrea das amostras, bem como os valores dos dois picos de fusão. Conforme Pluta et al. ${ }^{[37]}$, a região de temperatura de fusão tem direta correlação com o percentual de carga/fibra utilizada nas formulações. $\mathrm{O}$ processo de fusão torna-se mais complexo e dois picos de fusão são registrados nos diagramas de $\mathrm{DSC}^{[36,38]}$. Os dois picos de fusão $\left(\mathrm{Tm}_{1}\right.$ e $\mathrm{Tm}_{2}$ ) foram observados em praticamente todas as formulações avaliadas, inclusive naquela referente ao padrão, sem a presença da fibra. 
Todavia, tal fato também era esperado, haja vista que, segundo alguns autore ${ }^{[36,39]}$, tal evento possa ser atribuído ao rearranjo lamelar ocorrido durante a cristalização do PLA, isto é, ao efeito da fusão dos cristais originais (baixa temperatura de pico), recristalização e refusão dos cristais (alta temperatura de pico) durante a verificação de aquecimento; à existência de mais de uma estrutura de cristal; às condições de processamento ou distribuição de massa molar.

Esperar-se-ia que a adição de um agente compatibilizante pudesse impactar no decréscimo da $\mathrm{Tg}$ e no grau de cristalinidade $(\mathrm{Xc})$ do materia ${ }^{[38,40]}$. Entretanto, não houve uma diminuição pronunciada da $\mathrm{Tg}$, o que também poderia ser um indicativo da baixa interação fibra/matriz polimérica. Verifica-se, também, um declínio na temperatura de cristalização (Tc) quando adicionadas as cargas vegetais à blenda de PLA/TPU. Tal comportamento sugere que as cargas, igualmente, podem ter atuado como agente nucleante da matriz polimérica ${ }^{[41]}$.
No intuito de conhecer a estrutura/morfologia dos compósitos, realizaram-se análises de microscopia eletrônica de varredura, uma técnica valiosa de caracterização $^{[42]}$, cujos resultados são ilustrados pelas Figuras 6 e 7 referentes às amostras contendo $30 \%$ de fibra vegetal na presença ou ausência do agente compatibilizante. A Figura 6 está relacionada à formulação contendo $30 \%$ em massa de fibra de madeira, enquanto a Figura 7 é atribuída à formulação com teor de $30 \%$ em fibra de coco.

Analisando-se as micrografias de uma forma geral, observa-se a presença de vazios com consequente fraca adesão entre as fibras e a matriz polimérica, mesmo após a inclusão do anidrido maleico, indo ao encontro dos resultados obtidos através dos ensaios mecânicos, os quais apresentavam baixa interação fibra/matriz polimérica. Em particular, verifica-se um resultado mais pronunciado para os compostos contendo fibra de coco. O comportamento observado por essa amostra pode ser atribuído à característica higroscópica da fibra de $\operatorname{coco}^{[43]}$,

Tabela 2. Dados comparativos de diferentes composições com a blenda PLA/TPU com/sem agente de acoplamento na presença das fibras de madeira e coco.

\begin{tabular}{|c|c|c|c|c|c|c|c|c|c|}
\hline \multirow{2}{*}{ Formulações } & \multirow{2}{*}{$\operatorname{Tg}\left({ }^{\circ} \mathbf{C}\right)$} & \multirow{2}{*}{$\mathbf{T c}\left({ }^{\circ} \mathbf{C}\right)$} & \multirow{2}{*}{$\Delta \mathbf{H}_{\mathrm{c}}\left(\mathrm{Jg}^{-1}\right)$} & \multicolumn{2}{|c|}{$\operatorname{Tm}\left({ }^{\circ} \mathrm{C}\right)$} & \multirow{2}{*}{$\Delta \mathbf{H}_{\mathrm{m} 1}\left(\mathrm{Jg}^{-1}\right)$} & \multirow{2}{*}{$\Delta \mathbf{H}_{\mathrm{m} 2}\left(\mathrm{Jg}^{-1}\right)$} & \multirow{2}{*}{$\begin{array}{c}\Delta \mathbf{H}_{\text {mTotal }} \\
\left(\mathbf{J g}^{-1}\right)\end{array}$} & \multirow{2}{*}{ Xc $(\%)$} \\
\hline & & & & $\operatorname{Tm}_{1}\left({ }^{\circ} \mathbf{C}\right)$ & $\operatorname{Tm}_{2}\left({ }^{\circ} \mathbf{C}\right)$ & & & & \\
\hline PADRÃO & 56,90 & 111,01 & $-16,37$ & 148,47 & 155,80 & 2,91 & 6,92 & 9,83 & 40,20 \\
\hline FM10SA & 57,94 & 108,99 & $-13,54$ & 148,32 & 155,15 & 1,82 & 3,43 & 5,25 & 32,03 \\
\hline FM10CA & 56,35 & 102,84 & $-9,42$ & 144,65 & 153,47 & 1,32 & 7,26 & 8,58 & 31,97 \\
\hline FM20SA & 57,16 & 106,33 & $-13,74$ & 147,93 & 156,27 & 1,94 & 6,49 & 8,43 & 42,52 \\
\hline FM20CA & 57,42 & 104,83 & $-9,14$ & 145,82 & 154,14 & 1,34 & 6,03 & 7,37 & 33,16 \\
\hline FM30SA & 55,89 & 109,50 & $-10,55$ & 147,80 & 154,96 & 2,23 & 3,04 & 5,27 & 34,68 \\
\hline FM30CA & 54,99 & 105,13 & $-19,10$ & 146,41 & 155,05 & 2,26 & 8,75 & 11,01 & 69,57 \\
\hline FC10SA & 56,93 & 104,50 & $-8,11$ & 148,95 & 156,09 & 1,01 & 4,31 & 5,32 & 22,90 \\
\hline FC10CA & 53,80 & 96,60 & $-5,21$ & 153,85 & - & 17,18 & - & 17,18 & 39,75 \\
\hline FC20SA & 55,18 & 105,16 & $-8,67$ & 155,76 & - & 18,82 & - & 18,82 & 52,72 \\
\hline FC20CA & 55,26 & 102,31 & $-8,45$ & 144,95 & 154,40 & 0,70 & 9,10 & 9,80 & 36,65 \\
\hline FC30SA & 56,75 & 110,02 & $-11,86$ & 148,63 & 155,47 & 1,49 & 2,25 & 3,73 & 34,19 \\
\hline FC30CA & 55,71 & 105,50 & $-14,02$ & 146,79 & 155,26 & 2,02 & 8,27 & 10,29 & 56,16 \\
\hline
\end{tabular}

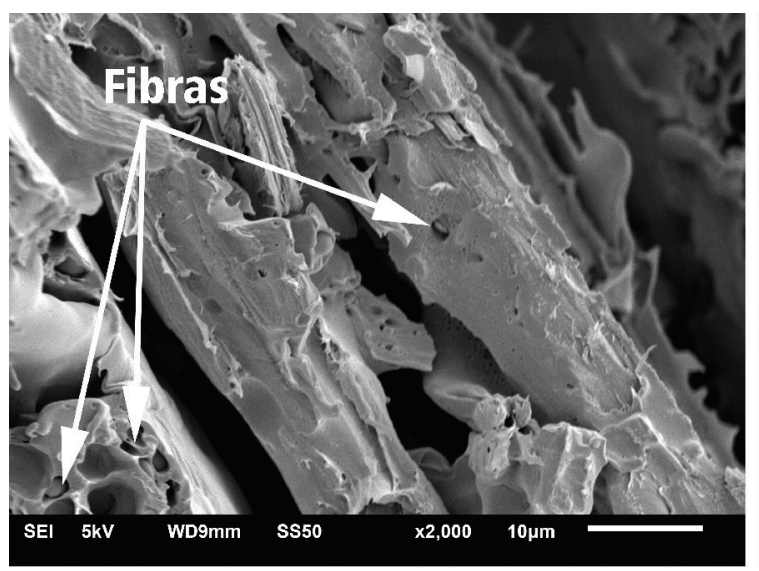

(a)

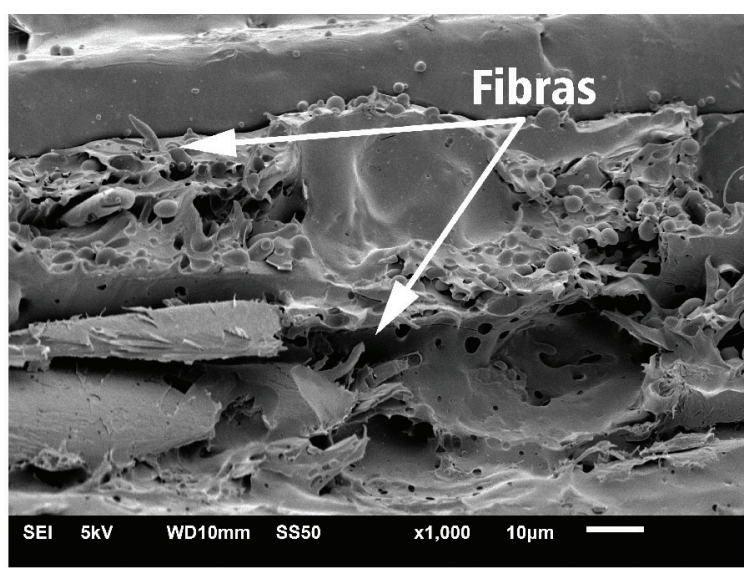

(b)

Figura 6. Micrografia dos compósitos contendo $30 \%$ em massa de fibra de madeira na ausência (a) ou na presença (b) presença de anidrido maleico, mostrando a superfície transversal dos mesmos. As setas em a) e b) mostram as fibras (ampliação de 2000x e 1000x, respectivamente). 


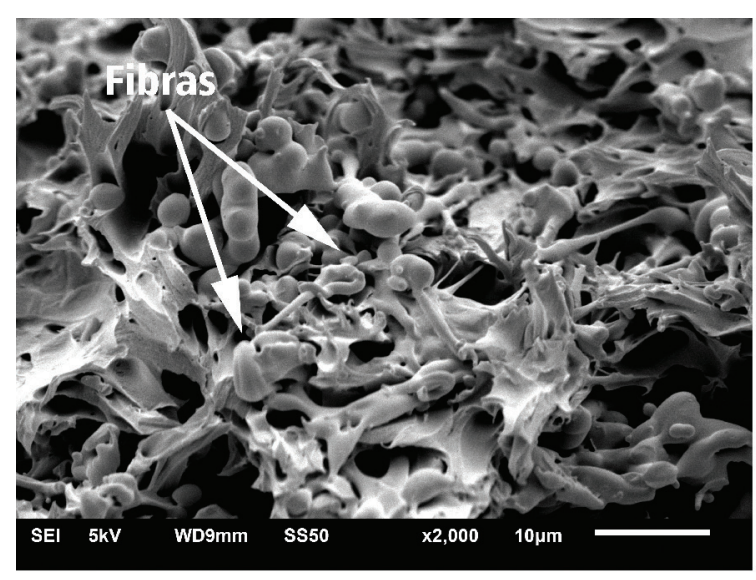

(a)

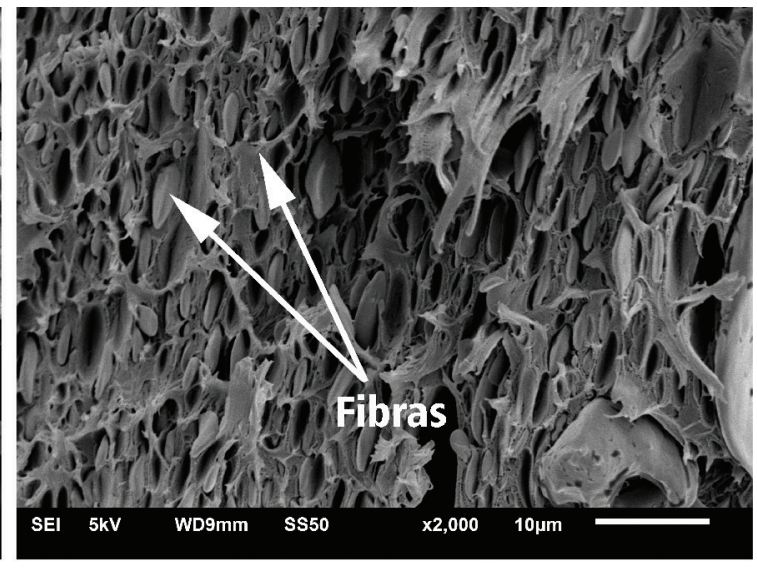

(b)

Figura 7. Micrografia dos compósitos contendo 30\% em massa de fibra de coco na ausência (a) ou na presença (b) presença de anidrido maleico, mostrando a superfície transversal dos mesmos. As setas em a) e b) mostram as fibras (ampliação de 2000x).

levando à baixa interação com a matriz polimérica. Nesse ponto, a fibra pode agir como um agente de separação na interface fibra/matriz, causando pontos de vazios na superfície do compósito, como verificado nas imagens, levando a falhas no mesmo, como o aparecimento de trincas na matriz ou mesmo o descolamento da fibra (debonding $)^{44}$.

\section{Conclusões}

Os biocompósitos da blenda de PLA/TPU reforçados com fibras de madeira apresentaram propriedades ligeiramente superiores quando comparadas aos biocompósitos da blenda de PLA/TPU com fibras de coco, utilizando-se as mesmas matrizes e mesmas condições de processamento. As diferenças encontradas entre as formulações podem ser atribuídas às propriedades mecânicas das fibras de madeira comparadas às das fibras de coco quando da adição destas à matriz. A dispersão da fibra de coco pode, também, ter influenciado a eficiência de reforço no compósito percebido pelo alto desvio padrão da maioria das análises. Todavia, quando se faz a comparação entre todas as formulações contendo fibras em relação àquelas sem a presença das mesmas, observa-se, em geral, queda das propriedades avaliadas para os compósitos PLA/TPU/fibras naturais com ou sem o agente de acoplamento, denotando baixa adesão interfacial entre a matriz (PLA/TPU) e as fibras.

Infere-se que os biocompósitos avaliados não atendem às exigências necessárias para utilizá-los em situações nas quais há necessidade de alto desempenho, como no setor automotivo, por exemplo. Contudo, a escolha adequada da fibra e da porcentagem desta na formulação do compósito final pode levar à confecção de produtos que, embora apresentem baixo desempenho, possam atender a necessidade de nichos específicos de mercados populares como o de decoração, viabilizando o uso de fibras vegetais em razão da redução de custo do compósito final.

\section{Agradecimentos}

Os autores agradecem à Artecola, à Merquinsa, à NatureWorks e ao Laboratório de Estudos Avançados em Materiais, da Universidade Feevale, pelas análises de MEV.

\section{Referências Bibliográficas}

1. Raquez, J. M.; Deléglise, M.; Lacrampe, M. F. \& Krawczak, P. - Prog. Polym. Sci., 35, p.487 (2010). http://dx.doi. org/10.1016/j.progpolymsci.2010.01.001

2. Cheung, H.; Ho, M.; Lau, K.; Cardona, F. \& Hui, D. - Compos. Part B Eng., 40, p.655 (2009). http://dx.doi. org/10.1016/j.compositesb.2009.04.014

3. Vilaseca, F.; Gonzalez, A. V.; Franco, P. J. H.; Pèlach, M. À.; López, J. P. \& Mutjé, P. - Bioresource Tech., 101, p.9264 (2010).

4. Graupner, N.; Herrmann, A. S. \& Müssig, J. - Compos. Part A Appl. Sci. Manuf., 40, p.810 (2009). http://dx.doi. org/10.1016/j.compositesa.2009.04.003

5. Sykacek, E.; Hrabalova, M.; Frech, H. \& Mundigler, N. - Compos. Part A Appl. Sci. Manuf., 40, p.1272 (2009). http://dx.doi.org/10.1016/j.compositesa.2009.05.023

6. John, M. J. \& Thomas, S. - Carbohyd. Polym., 71, p.343 (2008). http://dx.doi.org/10.1016/j.carbpol.2007.05.040

7. Jamshidian, M.; Tehrany, E. A.; Imran, M.; Jacquot, M. \& Desobry, S. - Compr. Rev. Food Sci. F., 9, p.552 (2010). http://dx.doi.org/10.1111/j.1541-4337.2010.00126.x

8. Paoli, M. A. - "Compósitos de Polímeros com Fibras Vegetais", in: 1 Workshop sobre Fibras Naturais, São Paulo (2010).

9. Paoli, M. A.; Spinacé, M. A. S.; Mano, B. I. S.; Araújo, J. R.; Janeiro, L. G. \& Bernardino, F.C. - "Substituição das Fibras Curtas de Vidro por Fibras Vegetais em Termoplásticos Reforçados" in: 1 Feira e Congresso Plastshow, p.1, São Paulo (2010). Relatório. 
10. Sanchez, E. M. S.; Cavani, C. S.; Leal, C. V. \& Sanchez, C. G. - Polímeros, 20, p.194 (2010). http://dx.doi.org/10.1590/ S0104-14282010005000034

11. Silva, R.; Haraguchi, S. K.; Muniz, E. C. \& Rubira, A. F. - Quím. Nova, 32, p.661 (2009). http://dx.doi. org/10.1590/S0100-40422009000300010

12. Velde, K. V. \& Kiekens, P. - Compos. Struct., 54, p.355 (2001).

13. Ashori, A. - Bioresource Technol., 99, p.4661 (2008). PMid:18068352. http://dx.doi.org/10.1016/j. biortech.2007.09.043

14. Shenoy, A. V. "Rheology of Filled Polymer Systems", Kluwer Academic Publishers, Dordrecht, p.475 (1999). http://dx.doi.org/10.1007/978-94-015-9213-0

15. Barreto, A. C.; Esmeraldo, M. A.; Rosa, D. S.; Fechine, P. B. A. \& Mazzetto, S. E. - Polym. Compos, 31, p.1928 (2010). http://dx.doi.org/10.1002/pc.20990

16. Barreto, A. C.; Júnior, A. E. C.; Rosa, D. S.; Barcellos, W. M.; Freire, F. N. A.; Fechine, P. B. A. \& Mazzetto, S. E. - J. Compos. Mater., 47, p.459 (2013). http://dx.doi. org/10.1177/0021998312441041

17. Morales, A. R.; Paiva, L. B.; Zattarelli, D. \& Guimarães, T. R. - Polímeros, 22, p.54 (2012). http://dx.doi.org/10.1590/ S0104-14282012005000004

18. Ferreira, K. R. M.; Leite, I. F.; Siqueira, A. S.; Raposo, C. M. O.; Carvalho, L. H. \& Silva, S. M. L. - Polímeros, 21, p.421 (2011). http://dx.doi.org/10.1590/S010414282011005000072

19. Gomes, A. S.; Barbosa, R. V. \& Soares, B. G. - Polímeros, 2, p.19 (1992).

20. Tedesco, A. "Polipropileno Graftizado com Metacrilato de Glicidila como Agente Compatibilizante em Blendas de PP e Nylon 6", Dissertação de Mestrado, Universidade Federal do Rio Grande do Sul, Porto Alegre (2001).

21. Santos, E. F.; Moresco, M.; Rosa, S. M. L. \& Nachtigall, S. M. B. - Polímeros, 20, p. 215 (2010). http://dx.doi. org/10.1590/S0104-14282010005000036

22. Lasprilla, A. J. R. - "Síntese do Poli(Ácido Láctico) a partir do Ácido Láctico para Aplicação Biomédica”, Dissertação de Mestrado, Universidade Estadual de Campinas, Campinas (2011). PMid:21756992.

23. Fiorio, R. - "Efeitos da Incorporação de Oligouretanos de Baixa Massa Molar nas Propriedades Térmicas, Mecânicas e Reológicas do Poliuretano Termoplástico", Dissertação de Mestrado, Universidade Federal do Rio Grande do Sul, Porto Alegre (2007)

24. Zhao, Y.; Qiu, J.; Feng, H.; Zhang, M.; Lei, L. \& Wu, X. - Chem. Eng. J., 173, p.659 (2011). http://dx.doi. org/10.1016/j.cej.2011.07.076

25. Barreto, A. C. H.; Costa, M. M.; Sombra, A. S. B.; Rosa, D. S.; Nascimento, R. F.; Mazzetto, S. E. \& Fechine, P. B. A. - J. Polym. Environ., 18, p.523 (2010). http://dx.doi. org/10.1007/s10924-010-0216-X

26. Satyanarayana, K. G.; Arizaga, G. G. C. \& Wypych, F. - Prog. Polym. Sci., 34, p.982 (2009). http://dx.doi. org/10.1016/j.progpolymsci.2008.12.002

27. Avella, M.; Buzarovska, A.; Errico, M. E.; Gentile, G. \& Grozdanov, A. - Materials, 2, p.911 (2009). http://dx.doi. org/10.3390/ma2030911
28. Bax, B. \& Müssig, J. - Compos. Sci. Technol., 68, p.1601 (2008). http://dx.doi.org/10.1016/j.compscitech.2008.01.004

29. Baillie, C. - "Green composites: polymer composites and the environment", CRC Press, Cambridge (2004).

30. Barreto, A. C. H.; Rosa, D. S.; Fechine, P. B. A. \& Mazzetto, S. E. - Compos. Part A Appl. Sci. Manu, 42, p.492 (2011). http://dx.doi.org/10.1016/j.compositesa.2011.01.008

31. Neto, F. L.; Pardini, L. C. - "Compósitos estruturais: ciência e tecnologia”, Edgar Blücher, São Paulo (2006).

32. Isoldi, A. B. G. - "Estudo do Efeito da Dose de Radioesterilização sobre as Propriedades do Poli(Tereftalato de Etileno) - PET - Reciclado", Dissertação de Mestrado, Instituto de Pesquisas Energéticas e Nucleares (Autarquia Associada à Universidade de São Paulo), Brasil (2003).

33. Santos, P. A.; Spinacé, M. A. S.; Fermoselli, K. K. G.; Paoli, M. A - Polímeros, 19, p.31 (2009).

34. Vianna, L. W.; Correa, A. C. \& Razzino, A. C. - Polímeros, 14, p.339 (2004)

35. Borsoi, C.; Scienza, L. C.; Zattera, A. J. \& Angrizani, C. G. - Polímeros, 21, p.271 (2011). http://dx.doi.org/10.1590/ S0104-14282011005000055

36. Way, C.; Dean, K.; Wu, D. Y.; Palombo, E. A. - J. Polym. Environ., 19, p.849 (2011). http://dx.doi.org/10.1007/ s10924-011-0361-x

37. Pluta, M.; Murariu, M.; Alexandre, M.; Galeski, A.; Dubois, P. - Polym. Degrad. Stabil., 93, p.925 (2008). http://dx.doi. org/10.1016/j.polymdegradstab.2008.02.001

38. Qin, L.; Qiu, J.; Liu, M.; Ding, S.; Shao, L.; Lü, S.; Zhang, G.; Zhao, Y.; Fu, X. - Chem. Eng. J., 162, p.772 (2011). http://dx.doi.org/10.1016/j.cej.2010.11.039

39. Hughes, J.; Thomas, R.; Byun, Y. - Carbohyd. Polym., 88, p.165 (2012). http://dx.doi.org/10.1016/j. carbpol.2011.11.078

40. Hwang, S. W.; Lee, S. B.; Lee, C. K.; Lee, J. Y.; Shim, J. K.; Selke, S. E. M.; Valdez, H. S.; Matuana, L.; Rubino, M. - Polym. Test., 31, p.333 (2012). http://dx.doi. org/10.1016/j.polymertesting.2011.12.005

41. Pilla, S.; Gong, S.; O’Neill, E.; Yang, L.; Rowell, R. M. - J. Appl. Polym. Sci., 111, p.37 (2009). http://dx.doi. org/10.1002/app.28860

42. Marinelli, A. L.; Monteiro, M. R.; Ambrósio, J. D.; Branciforti, M. C.; Kobayashi, M.; Nobre, A. D. - Polímeros, 18, p.92 (2008). http://dx.doi.org/10.1590/ S0104-14282008000200005

43. Gonçalves, J. A. V. - "Compostos à Base de Resina Epóxi Reforçados com Fibra de Coco", Dissertação de Mestrado, Universidade Federal de Sergipe, São Cristovão (2010).

44. Razera, I. A. T. - "Fibras Lignocelulósicas como Agente de Reforço de Compósitos de Matriz Fenólica e Lignofenólica”, Tese de Doutorado, Universidade de São Paulo, São Carlos (2006).

Enviado: 29/11/12

Reenviado: 03/05/13 Aceito: 10/06/13 